\title{
Geographiedidaktische Überlegungen zu Basiskonzepten des Unterrichtsfachs GWK unter kritischem Bezug auf sozial-kon- struktivistische Raumkonzepte und digitale Geomedien - ein Diskussionsbeitrag
}

\author{
*angela.hof@sbg.ac.at, Fachbereich für Geographie \& Geologie, Universität Salzburg \\ "*stefanie.huertgen@sbg.ac.at, Fachbereich für Geographie \& Geologie, Universität Salzburg \\ ***sandra.stieger@sbg.ac.at, Fachbereich für Geographie \& Geologie, Universität Salzburg
}

eingereicht am: 28.01.2016, akzeptiert am: 18.04.2016

\begin{abstract}
Der vorliegende Beitrag hat das Ziel, die für den neuen Geographie- und Wirtschaftskundelehrplan der AHS Oberstufe entwickelten Basiskonzepte, insbesondere das vorgeschlagene Basiskonzept: Raumkonstruktion und Raumkonzepte, mit grundlegenden Diskussionen zur Integration von raumtheoretischen Herangehensweisen im Unterricht zu verbinden. Wir hinterfragen insbesondere die Anschlussfähigkeit von sozialkonstruktivistischen Theorieangeboten einerseits (Werlen 2015) und dem Einsatz digitaler Geomedien im Unterricht andererseits. Resultat unserer Überlegungen ist eine doppelte Kritik dieser sozialkonstruktivistischen, zugespitzt auf „Entterritorialisierung“ abzielenden aktuellen Raum-Diskussion: erstens ist nach unserer Ansicht das metrische Raumkonzept inhärenter Bestandteil sowohl von Geomedien wie auch von realer Lebenspraxis, d. h. es ist in den Präkonzepten der Schüler/innen verankert; zweitens betonen wir, dass die „Entterritorialisierung" bzw. das „Verschwinden der Ferne“ (ebd.) Gefahr läuft, die multiscalare Dimension jeglicher sozialer Praxen und damit insbesondere auch bestehende gesellschaftliche Ungleichheitsstrukturen, in denen gehandelt wird, aus dem Blick zu verlieren. Wir führen unsere Überlegungen in einer Skizze für einen konkreten Unterrichtsentwurf zusammen, um den praktischen Gehalt des Diskussionsbeitrags zu verdeutlichen.
\end{abstract}

Keywords: Basiskonzepte, Unterricht, Theorie, Raum, GIS

\section{Didactic considerations for basic concepts of geography teaching, with critical reference to social constructivist concepts of space and to digital geomedia - a contribution to the debate}

The main objective of this paper is to bring together discourses and geographical theory about space (Werlen 2015) with relatively new inspirations concerning the proposed introduction of so-called basic concepts into the geography curricula in secondary education in Austria. Considering this challenge, we outline and discuss, on the one hand, to which extent social constructivist theory can be connected to and made fruitful for treating basic concepts in teaching, and, on the other, we extend this same perspective to digital geomedia in geography teaching. As a result, we present a twofold critique of this social constructivist theory and its way of pinning down the current debate on space on 'de-territorialization' as a main theme. We argue that this falls short because, first, the metric concept of absolute space is an inherent concept of both geomedia and preconcepts of students, and, second, because 'de-territorialization' and 'disappearing distance' (ibid.) run the risk of neglecting the multiscalar dimension of social realities and the diversity of uneven developments and contexts in which social practices are embedded. These reflections are translated into a tangible lesson design that demonstrates the practical content of our considerations.

Keywords: basic concepts, teaching, theory, space, GIS 


\section{Kurzeinleitung und Aufbau des Diskussi- onsbeitrags}

Der vorliegende Beitrag greift eine beginnende geographiedidaktische Diskussion und Forschung zur Integration von grundlegenden raumtheoretischen Herangehensweisen und sogenannten Basiskonzepten in den Lehrplan GWK auf (Abschnitt 2). Wir diskutieren die mögliche Bedeutung und Anschlussfähigkeit des Basiskonzepts „Raumkonstruktion und Raumkonzepte" vor dem Hintergrund ausgewählter raumtheoretischer Paradigmen. Konzeptionell und theoretisch knüpft dieser Beitrag an die in den letzten Jahren in der Geographie und den Kultur- und Sozialwissenschaften breit geführte Debatte zur Neuthematisierung des Raumes an (Abschnitt 3). Wir fragen uns, in welcher Weise sind innovative Ansätze und Basiskonzepte im Schulkontext kompatibel zu ausgewählten fachwissenschaftlichen Theorieangeboten. Dabei betrachten wir insbesondere die Anschlussfähigkeit von Basiskonzepten zu sozialkonstruktivistischen Theorieangeboten einerseits (Werlen 2015) und digitalen Geomedien andererseits. Anschlussfähigkeit kann, das ist unsere These, vor allem dann gewährleistet werden, wenn erstens metrische Raumkonzepte und zweitens multiscalare Perspektiven in die geomediale Betrachtung integriert werden. Wir skizzieren einen Vorschlag zur Operationalisierung durch einen konkreten Unterrichtsentwurf, um den praktischen Gehalt unserer Überlegungen zu verdeutlichen (Abschnitt 4).

Für das Unterrichtsfach Geographie stellt dieser Beitrag also dar, wie fachwissenschaftliche Raumkonstruktionen variiert und erweitert werden sollten, um mit entsprechend vermuteten metrischen ${ }^{1}$ wie auch multiscalaren ${ }^{2}$ Präkonzepten der Schüler/innen arbeiten zu können. Bezogen auf die Präkonzepte ist es ein explizites Lernziel, den Schülerinnen und Schülern einen aktiven Umgang mit und eine Hinterfragung der wissenschaftlichen Theorienangebote zu ermöglichen. Die sozial-konstruktivistische Perspektive erweitert das Verständnis der Schüler/innen dahingehend, dass Raum immer auch leiblich und physisch-materiell konkret ist und durch kommunikative, aber eben auch durch andere soziale Praxen wie Wahrnehmungs-, Denk- und Handlungsschemata im Kontext bestehender gesellschaftlicher Ungleichheitsstrukturen hervorgebracht wird.

z. B. die Vorstellung von Räumen als Container, die durch Distanz- und Lagebeziehungen charakterisiert sind.

2 z. B. Denken in verschiedenen Maßstabsebenen, insbesondere auch die Berücksichtigung von sozialen Ungleichheitsstrukturen.

\section{Anlass für die geographiedidaktischen Überlegungen und den Diskussionsbei- trag: Geplante Integration von Basiskon- zepten in den neuen semestrierten Lehr- plan der Sekundarstufe II AHS}

In der Konzeptvorlage (2014) der ministeriellen Arbeitsgruppe für den geplanten semestrierten Lehrplan für die Sekundarstufe II der Allgemein höherbildenden Schulen (AHS) in Österreich werden neben den Kompetenzen für das Unterrichtsfach Geographie und Wirtschaftskunde (UF GWK), erstmals auch sogenannte Basiskonzepte ausgewiesen. ${ }^{3}$ Ausgangspunkt für das Verständnis der Basiskonzepte ist der Begriff des Konzepts. Ein Konzept kann als eine Vorstellung oder als ein Denkakt definiert werden, der zum Verständnis der eigenen Lebenswelt beiträgt (Hellmuth 2014; Uhlenwinkel 2013).

Für den Unterricht sollten Basiskonzepte als Gerüst verstanden werden, das innerhalb eines strukturierten, kumulativen und langfristigen Lern- und Lehrprozesses entsteht. In diesem Gerüst werden Begriffe, Theorien und Modellvorstellungen zusammengeführt und miteinander vernetzt. Dabei sollten die Schüler/innen erkennen, dass Basiskonzepte in unterschiedlichen, für sie unbekannten Sachverhalten immer wiederkehren. Das dient einerseits zum Aufbau langfristiger Wissensstrukturen, andererseits können dadurch komplexe Sachverhalte seitens der Schüler/innen bewältigt werden (Mehren \& Mehren 2015, 69).

In diesem Zusammenhang stellt sich nun die Frage, welche Konzepte des Faches GWK als Basiskonzepte ausgewiesen werden sollten. Unbestritten musste die österreichische Arbeitsgruppe eine Auswahl treffen, da, wie beispielsweise Hellmuth anmerkt, Basiskonzepte Konstrukte sind und „[...] nicht zuletzt durch den Fortschritt fachwissenschaftlicher Forschungen - keinen Absolutheitsanspruch erheben und niemals alle Teilkonzepte berücksichtigt werden können" (Hellmuth 2014, 241 f.) ${ }^{4}$. Zudem tragen Basiskonzepte dazu bei, eine stärkere Anbindung des Unterrichtsfachs an aktuelle nomothetische und idiographische Konzepte der Fachwissenschaften zu fördern und die Kompetenzorientierung der Schüler/innen

\footnotetext{
Die 13 vorgeschlagenen Basiskonzepte sind: Raumkonstruktion und Raumkonzepte; Regionalisierung und Zonierung; Diversität und Disparität; Maßstäblichkeit; Wahrnehmung und Darstellung; Nachhaltigkeit und Lebensqualität; Interessen, Konflikte und Macht; Arbeit, Produktion und Konsum; Märkte, Regulierung und Deregulierung; Wachstum und Krise; Mensch-UmweltBeziehungen; Geoökosysteme; Kontingenz (Hinsch et al. 2014).

4 Laut der österreichischen Arbeitsgruppe „stellen [Basiskonzepte] den fachlichen Kern der geographischen und ökonomischen Bildung dar und konkretisieren, was aus der aktuellen Sicht der Fachwissenschaft(en) mit den jeweiligen Lehrplanformulierungen gemeint sein kann" (Hinsch et al. 2014, 52).
} 
zu unterstützen ${ }^{5}$. Zusätzlich sind Basiskonzepte eine inhaltliche und methodische Ergänzung der Lernziele und sollen die Schüler/innen zu reflektiertem Handeln befähigen. Folglich wird dem Begriff der „Basiskonzepte“ das Adjektiv "handlungsorientiert" vorangestellt (ebd.). Nach der österreichischen Definition der "handlungsorientierten Basiskonzepte“ wird insbesondere die Verknüpfung des Unterrichtsfaches mit den Fachwissenschaften angestrebt. Hingegen wird in den deutschen Bildungsstandards, die für alle Unterrichtsfächer gelten, hervorgehoben, dass Basiskonzepte einen grundlegenden Beitrag zum Weltverständnis für das jeweilige Unterrichtsfach leisten müssen (Sander 2010). Für einen solchen geforderten Beitrag zu einem "grundlegenden Weltverständnis“ der Schüler/ innen, ist es aus fachdidaktischer Sicht unumgänglich, einen Bezug zu den Präkonzepten, also den subjektiven Vorstellungen, der Schüler/innen herzustellen, die dann im Unterricht aufgegriffen und mit denen weiter gearbeitet wird (Stieger 2016). Durch einen Vergleich der Präkonzepte der Schüler/innen mit ausgewählten Basiskonzepten der Fachwissenschaft können also Anknüpfungspunkte gefunden werden, die es zulassen, dass aktuelle Konzeptionen der Fachwissenschaften in den Unterricht eingebunden werden. Keinesfalls sollten Basiskonzepte eines Unterrichtsfaches eine eindimensionale Sichtweise zulassen, sondern in Form eines multiperspektivischen Ansatzes gedacht sein, der möglicherweise konträre Vorstellungen der Fachwissenschaft gegenüberstellt und somit die wissenschaftlichen Auseinandersetzungen in einem Fach zum Thema gemacht werden können (Sander 2010).

Für den Beitrag wollen wir uns im Folgenden auf jenes Basiskonzept konzentrieren, das den Raumbegriff und das Raumverständnis thematisiert. In der österreichischen Vorlage für den neuen semestrierten Lehrplan wird empfohlen, im Unterricht auf mindestens drei unterschiedliche Raumkonzepte Bezug zu nehmen: auf das klassische Raumkonzept (für naturwissenschaftliche Analyse und kartographische Kommunikation), auf den (subjektiven) Wahrnehmungsraum (als Grundlage raumbezogener Images und Identitäten sowie als Bezugsgröße für handlungsleitende Entscheidungen) und auf das Konzept der interessengeleiteten Raumkonstruktionen (innerhalb

\footnotetext{
$\mathrm{Zu}$ den nomothetischen Fachkonzepten für das UF GWK zählen beispielsweise Produktlebenszyklus-Theorie, Standortfaktoren und Clustertheorien, Nachhaltigkeit als normatives Leitbild gesellschaftlichen Handelns, das Ökosystemkonzept, Kategorisierungen des Formenschatzes der Landoberfläche und der Syndromansatz, zum Beispiel zur Erklärung von Umweltproblemen und -konflikten. Der Syndromansatz vermittelt mit der Betonung der Fallstudie und einer pars pro toto Perspektive deutlich zu idiographischen Konzepten, die von Fallstudien Verallgemeinerungen entwickeln.
}

gesellschaftlicher Entscheidungs- und Aushandlungsprozesse) (Hinsch et al. 2014, 52). Diese Empfehlungen stützen sich auf jene Raumkonzepte, die schon von Wardenga (2002) im Zuge der Lehrplanarbeit für Geographie in Deutschland (Curriculum 2000+) diskutiert wurden. Hier plädiert Wardenga dafür, dass für einen Geographieunterricht, der sich den Herausforderungen des 21. Jahrhunderts stellen möchte, konstruktivistische Raumkonzepte unumgänglich sind (Wardenga 2002). Auch in der österreichischen Debatte wird hervorgehoben, dass Raumkonzepte den Schülerinnen und Schülern als ,[...] Produkte bestimmter Fragestellungen, bestimmter Betrachtungsansätze und auch Machtstrukturen [...]" (Sitte 2004, 47) bewusst gemacht werden sollten. Im aktuellen Vorschlag des Lehrplans wird diese Diskussion wieder aufgenommen, wobei im Unterschied zu den vier vorgeschlagenen Raumkonzepten von Wardenga (2002), zwei dieser Raumkonzepte, nämlich der „Raum als Container" und der „Raum als System von Lagebeziehungen und Reichweiten", zusammengefasst wurden zum „klassischen absoluten Raumkonzept“ (Hinsch et al. 2014, 52). Wir haben es also in der Debatte um raumbezogene Basiskonzepte für den Schulunterricht mit einem Spannungsverhältnis in der Relation von „absolutem“ oder metrischen und „sozialkonstruktivistischem" Raumverständnis zu tun. Um dieses Spannungsverhältnis, und wiederum dessen Verhältnis zu GIS, geht es uns im vorliegenden Artikel. Entsprechend werden wir im Folgenden schlaglichtartig auf die metrische wie die sozialkonstruktivistische Perspektive eingehen - und zwar von vornherein in Bezug auf die zu diskutierende, auf digitale Geomedien ausgerichtete Unterrichtspraxis. Dabei werden wir - insbesondere unter Bezugnahme auf Werlens (2015) sozial-konstruktivistischen Zugang - erstens die Fortexistenz des klassischen, absoluten Raumkonzepts thematisieren, die insbesondere mit Bezug auf den großen Stellenwert der Geoinformationstechnologien und der digitalen Geomedien deutlich wird, zweitens argumentieren, warum es einer Fortexistenz einer multiscalaren Betrachtungsweise bedarf, das heißt, warum konzeptionell weitere scales, und nicht nur die globale, aufrecht zu erhalten sind, drittens und damit zusammenhängend darlegen, warum die Werlen'sche (2015) Annahme der Entterritorialisierung zu kurz greift, und viertens thematisieren, warum aufgrund der metrischen Begrenztheit von GIS diese nicht problemlos als Instrument für die Auseinandersetzung mit sozial-konstruktivistischen Raumkonzepten verwendet werden können. 


\section{Herstellung und Diskussion von fachli- chen Bezügen zum Basiskonzept „Raum- konstruktion und Raumkonzepte“}

Nach wie vor gehört zu den fachwissenschaftlichen Raumkonzepten das Verständnis, dass Raum auch ein System von Lage- und Distanzbeziehungen ist. Allen Geoinformationstechnologien liegt ein topologisches Raumverständnis zugrunde, das geographiedidaktisch für nachhaltigere Lernerfolge in punkto räumlicher Orientierungskompetenz und topologischer Zusammenhänge (physisch-) geographischer Phänomene eingesetzt werden kann (Hof 2014; Kisser 2016). Im vorgeschlagenen Basiskonzept und den Empfehlungen zur Lehrplanarbeit (vgl. Abschnitt 2) ist die Forderung nach einer umfassenden Behandlung des Raumbegriffs im Geographieunterricht inbegriffen diese Forderung impliziert, Schüler/innen eine fachwissenschaftliche Bandbreite von Raumkonzepten aufzuzeigen. Wir unterstellen, dass ein zeitgemäßer Geographieunterricht zugleich digitale Geomedien (vgl. Siegmund \& Michel 2013) einsetzt und damit nicht umhinkommt, das Spannungsfeld zwischen diesen Repräsentationen und anderen Raumkonzepten und der damit verbundenen Theorieangebote zu berücksichtigen - ja dieses Spannungsfeld könnte sogar selbst zum Unterrichtsthema werden. Die Kombination von Geomedien einerseits und konstruktivistischen Raumbildern andererseits ist zugegebenermaßen sperrig, und steht wie im Folgenden kurz ausgeführt gewissermaßen quer zu einigen Denktraditionen, aber gerade dies macht die hier vorgeschlagene Kombination so wertvoll. Wir zeigen eine skizzenhafte Übersicht aktueller einflussreicher fachlicher Theorieangebote auf. Davon ausgehend, wurden zwei Theorien ausgewählt, die thesenartig in ihren Möglichkeiten und Begrenztheiten als Unterrichtsthema zusammengefasst werden.

\subsection{Fachwissenschaftliche Raumkonzepte und Theorieangebote und ihre Anschlussfähigkeit zum vorgeschlagenen Raumkonzept - ein Kurzüberblick}

Im multiparadigmatischen Brückenfach Geographie (Dürr \& Zepp 2012) sind neben der Systemtheorie (Egner 2006; Lippuner 2010) und Hybridmodellen (Lippuner 2009) insbesondere sozial-ökologische (Fischer-Kowalski \& Erb 2006; Wardenga \& Weichhart 2006) und sozial-konstruktivistische Theorieangebote (Werlen \& Lippuner 2011) vielfach rezipiert und für das Fach und seine Raumkonzepte fruchtbar gemacht worden.

Das erste einflussreiche Theorieangebot ist also das sozial-ökologische Interaktionsmodell von Gesell- schaft und Natur. Gesellschaft steht durch ständigen energetisch-materiellen Austausch in einem metabolischen Zusammenhang mit verschiedenen natürlichen Systemen und beeinflusst diese in bestimmter Weise, ist also in der Lage, materielle Realitäten raum-zeitlich zu verändern (Fischer-Kowalski \& Erb 2006; 37 ff.). Die Soziale Ökologie stellt recht pragmatisch verschiedene Wissenschaftstraditionen und deren elaborierte Methoden in den Dienst neuer interdisziplinärer Aufgaben und widmet sich in erster Linie den räumlichen Dimensionen gesellschaftlicher Naturverhältnisse. Ein eigenes Raumkonzept für gesellschaftlichen Metabolismus im Raum ist dabei nicht theorieleitend, sondern die konkrete räumliche Verbindung zwischen Sozialem und Naturalem muss unter variierenden Maßstabsbezügen und Skalenebenen erst konstruiert werden - sie ist nicht einfach durch das räumliche Anordnungsmuster einer Landschaft vorgegeben (Fischer-Kowalski \& Erb 2006, 43 ff.). Dieser Ansatz ist jedoch weniger sozialkonstruktivistisch, als dieses Postulat vermuten lässt, denn die Fokussierung auf Stoff- und Materialflüsse, auf den Zugriff auf Ressourcen und deren gesellschaftliche Aneignung durch Arbeit und Produktion geschieht vorwiegend durch Festlegung spezifischer Raumeinheiten, auf die sich die jeweiligen Bilanzierungen beziehen.

Die handlungstheoretisch orientierte Sozialgeographie wiederum beschäftigt sich mit geographischen Praktiken „des alltäglichen Geographie-Machens“ (Werlen \& Lippuner 2011, 688), wobei der Begriff des Handelns die Verknüpfung von physisch-materiellen Gegebenheiten und der sozialen Welt leistet, die in der klassischen Geographie im Landschaftsbegriff und im Raumbegriff aufgehoben war (Weichhart 2011). Werlen $(2015,91)$ spricht von räumlichen und zeitlichen Bezügen als sozial-kulturelle Gestaltungsmittel, von deren Verfügbarkeit wesentlich die Vermögensgrade des Handelns über Distanz abhängen, welche die Konstitution und Etablierung gesellschaftlicher Raumverhältnisse maßgeblich bestimmen. Akteure setzen ihre Wirklichkeit in Bezug zu Raum und Zeit und gesellschaftliche Raumverhältnisse werden wesentlich dadurch bestimmt, in welchem Ausmaß räumliche Konstellationen handlungsrelevanter physisch-materieller Gegebenheiten bewältigt werden können.

Beide hier schlaglichtartig betrachteten theoretischen Perspektiven stehen nun allerdings in einem gewissen Spannungsverhältnis zu GIS, denn beide Debatten relativieren das oben genannte, klassischabsolute Raumkonzept - während GIS allerdings notwendigerweise mit eben diesem klassischen Raumkonzept arbeitet: In der geographiedidaktischen Diskussion (vgl. Siegmund \& Michel 2013; Schulze 2015) werden die Begriffe Geomedien, Geo- 
information und Geokommunikation als Teilaspekte digitaler Geomedien verwendet, deren Hauptkategorien digitale Globen (zum Beispiel Google Earth) Geographische Informationssysteme (GIS) und die Fernerkundung (Luft- und Satellitenbilder) sind. „Digitale Geomedien stellen digital codierte raumbezogene Daten über geographisch relevante Sachverhalte dar sowie die zu deren Verwaltung, Analyse und Visualisierung notwendigen technischen Geräte und Software" (Siegmund \& Michel 2013, 53). Schulze (2015) spricht von multimedialen Repräsentationen erdräumlicher Informationen. Digitale Geomedien basieren auf metrischen, kartesischen Raumkonzepten und repräsentieren und visualisieren erdräumliche Informationen in solchen Datenmodellen. Geoinformation beinhaltet grundsätzlich die eindeutig räumlich und zeitlich zugeordneten Merkmalsausprägungen von Entitäten auf der Erdoberfläche. Bereits diese Beschreibung orientiert sich an der Technologie, auf der Geomedien basieren: der computerbasierten, formallogischen Eingabe, Verarbeitung und Ausgabe von Geodaten.

Soll also das raumbezogene Basiskonzept im Unterricht fruchtbar behandelt werden, so unser Argument, dann geht es nicht um die (Schein-)Alternative sozialkonstruktivistisches oder absolutes Raumkonzept, sondern um die Art und Weise der Verbindung von beiden. Dafür allerdings ist eine genaue Verständigung darüber, wie die soziale Konstruktion von Räumen verstanden werden soll, notwendig, wie wir am Beispiel ausgewählter jüngerer Arbeiten von Benno Werlen (2015) im Folgenden darlegen wollen.

In der sozialgeographischen Diskussion radikalisiert Werlen eine sozialkonstruktivistische Herangehensweise an „Raum“. Mit seiner Konzeption von Raum als „Reflexionsbegriff“" (Werlen \& Weingarten 2013, 75) bzw. als „gesellschaftlichen Raumverhältnisse" (Werlen 2015, 85) will er das Räumliche von jeglichen Vorstellungen den sozialen Handlungen vorgängiger Beschaffenheit befreien und konsequent an soziales Handeln/Tätigsein (agency) binden. Damit geht Werlen auch über andere gesellschaftlichsoziale Definitionen von Raum hinaus, bspw. über die Definition von Löw, die Raum auffasst als relationales Element unter sozialen Elementen (Gütern und Lebewesen) an Orten (Löw 2001; s.a. Weidenhaus 2015). Werlen dagegen argumentiert gegen jegliches „Ansich-sein-Könnens" von Dingen (Werlen \& Weingarten 2013 , 77) und lehnt nicht nur eine dem Handeln vorgängige physische (Entstehungs-)Beschaffenheit, sondern überhaupt jegliche Vorstellungen einer vom Handeln gedanklich abgelösten „Materialität des Raums" ab (ebd., 75). Stattdessen gehe es - gegen "die traditionelle Geographie der Räume und Orte“ (Werlen 2015, 84) - um die soziale Hervorbringung „sinnhafter geographischer Wirklichkeiten“ in den alltäglichen Praxen der handelnden Subjekte (ebd.: 83 ff.; Werlen 2015, 88 ff.). Werlen schlägt vor,

Raum als formal-klassifikatorischen Begriff zu verstehen, der weder als materiell, relational noch ideell bestimmt werden kann. Er ist somit weder Gegenstand noch Wirkkraft, sondern ein Modus der Wirklichkeitskonstitution, der im Wesentlichen auf die Ausgedehntheit materieller Objekte, inklusive dem des menschlichen Körpers, sowie deren gegenseitige Ausschließlichkeit im Rahmen der Gleichzeitigkeit rekurriert (Werlen 2015: 89).

Diese radikale „Verabschiedung“ (Werlen 2015, 88) von Vorstellungen eines Handelns im Raum (der damit dem Handeln vorgängig gedacht sei und letztlich auf eine Containerisierung von Raum hinauslaufe) (ebd.), zugunsten einer „Fokussierung auf soziale Praktiken“ die Raum hervorbringen, enthält eine Reihe von wertvollen theoretischen Anregungen. Beispielsweise gilt es mit diesem Ansatz, alle Formen von Räumlichkeit als theoretisch zugänglich zu fassen (und nicht als vortheoretischen und damit unreflektierten "Container", vgl. Beck \& Grande 2004; Amelina et al. 2012) - vor allem aber ließen sich die unterschiedlichen sozialen Herstellungsweisen von Raum als Auseinandersetzung mit unterschiedlichen Macht- und Ressourcenausstattungen thematisieren.

Werlen bezieht sich in der Tat auch zur Begründung seines Ansatzes explizit auf hierarchische Machtverhältnisse bei der Herstellung von Raum (ebd., 84) - allerdings, und dies ist durchaus irritierend, spielen die sozialen und machtbezogenen Verschiedenheiten dann im Weiteren keine Rolle mehr. Vielmehr verfolgt Werlen nun eine weit weniger deutlich ausgewiesene normative These die besagt, dass gesellschaftliche Entwicklung durch das „Verschwinden der Ferne“ gekennzeichnet sei. Entwicklung ist hiernach prinzipiell ein Prozess der räumlichen „Ausdehnung“ und „Extension". Er macht dies an der Überwindung räumlicher Grenzen im Laufe der Geschichte wie bspw. der Herausbildung nationaler Gestaltungen des Politischen (und der Überwindung einzelner Dörfer und Ländereien) bis zur heutigen Globalisierung fest, wobei er letztere als Vorgang der „Entterritorialisierung“ fasst. Die Raumbezüge seien „offensichtlich in immer mehr Lebensbereichen in Auflösung begriffen " (Werlen 2015, 87). Dieser Prozess kulminiert nach Werlen in nichts weniger als einer Revolutionierung ,alter" Raumbezüge:

Mit dem "Verschwinden der Ferne" sind für zahlreiche Lebensbereiche und Handlungspraktiken unterschiedlichste Implikationen verbunden, womit eine große Zahl von etablierten Routinen und unhinterfragten Einschätzungen, Bewertungen etc. 
ihre Gültigkeit zum Teil in dramatischer Form einbüßen. [Es entstehe] die Aufhebung des Konnexes von Nähe und Vertrautheit bzw. Ferne und Unvertrautheit"(Werlen 2015: 90).

Diese prinzipielle These der „Entterritorialisierung“ ist darum verblüffend und auch kritik-provozierend, weil sie die insbesondere in der Geographie, und nicht zuletzt von Werlen selbst, entwickelte Perspektive einer Multiscalarität von Globalisierung, also der „Glokalisierung" (vgl. Robertson 1998; Swyngedouw 2004) nicht mehr fortführt. Die jüngeren ökonomischen und sozialen Umbrüche bedeuten hiernach gerade nicht nur Ausdehnung, sondern damit zusammenhängend auch (Re-)Lokalisierung (vgl. Kern 1994). Entsprechend wird insbesondere aus wirtschaftsgeographischer Sicht dafür plädiert, Globalisierung nicht einfach als Überwindung des Lokalen zu begreifen, sondern als dessen (durchaus permanente) Neukonfiguration (Brenner 2004; s. Beiträge in Belina \& Michel 2007). Werlen deutet diese Problematik zwar als Frage der "multiplen Ausrichtungspotenziale“ an, wonach auch lokale und regionale Phänomene nun vor dem Hintergrund der neuen Dimension globaler Extension neu interpretiert würden (Werlen 2015, 87), im Gesamtkontext der Darstellung allerdings verflüchtigen sich die lokalen, regionalen etc. scales im Zuge der Auseinandersetzung mit „Entterritorialisierung“. Dies umso mehr, als Werlen - vergleichbar dem Beck'schen „Kosmopolitismus“-Ansatz (Beck \& Grande 2004) - die nicht-globalen Scales vor allem in ihrer konservativen bzw. gleich ganz reaktionären Ausrichtung thematisiert: beispielsweise als nationalistische Naturalisierung bis hin zum „Blut-und-Boden-Theorem“ (Werlen 2015, 88) - während „Extension“ der (Spät-) Moderne und den ihr zugehörigen Lebensweisen zugehörig scheint.

Demgegenüber betonen die oben aufgeführten Ansätze weit expliziter die Notwendigkeit, die unterschiedlichen sozialen Ebenen als Gleichzeitigkeit zu denken. Statt eine sozial-räumliche Ebene gegen die andere - im Sinne sogar eines „Verschwindens“ oder einer tendenziellen Charakterisierung als "modern“ hier und "traditionell-konservativ" da - auszuspielen, ginge es darum, die Art und Weise des Zusammenwirkens der Reproduktion im Prinzip aller sozialer Scales in ihrer Unterschiedlichkeit zu erfassen. Methodologisch wäre also die Frage nach der Art und Weise der Herstellung von immer schon multiscalar gedachten Praxen prioritär (vgl. Herod 2011; s.a. Hürtgen 2015). Insbesondere sind dabei an ungleiche Ressourcen zur Gestaltung von Räumlichkeiten, aber auch zur Überwindung ,absoluter “ räumlicher Distanzen zu denken. Zugespitzt formuliert könnte sich die Kommunikation auf globaler Ebene für vie- le Menschen vor allem oder ausschließlich als virtuelle darstellen, während die eigene leiblich-materielle Existenz „vor Ort" verbleibt - während für diejenigen mit entsprechenden Ressourcen die Überwindung auch größerer Distanzen für kurze Zeit weit weniger problematisch sein dürften. Beide Male sind sowohl lokale, wie extensivierte Scales von Bedeutung, aber in unterschiedlicher Weise. Entsprechend gilt es an die Perspektive der Multiscalarität im eigenen Beitrag anzuschließen. Während Werlen (2015) letztlich danach fragt, welche Möglichkeiten den sozialen Subjekten gegeben sind, sich in einer "Welt der Ausgedehntheit" zu orientieren und sich diese anzueignen, lässt sich mit einer offensiv-multiscalaren Perspektive untersuchen, wer (sozial) auf welche Weise in der Lage ist, räumliche Grenzen zu überwinden und immer zugleich auch keineswegs sich auflösende räumliche Nahbeziehungen zu strukturieren. Mit Erik Swyngedouw ließe sich so - offener - nach "scale capabilities of individuals and social groups" fragen (vgl. Swyngedouw 2004, 34), und nicht nur oder hauptsächlich nach dem Vermögen in Bezug auf eine Scale, die räumliche Fernbeziehung. Generell ginge es mit Werlen wie mit Swyngedouw um die „verfügbaren Gestaltungsmittel der räumlichen und zeitlichen Bezüge" (Werlen 2015), aber eben sowohl was den Nah-, als auch was den Fernraum betrifft.

Um es kurz zusammenzufassen: Das Spannungsverhältnis von Raumbegriff und Geomedien ist ein mehrfaches: weder können der klassische absolute Raumbegriff verabschiedet, noch können aktuelle sozialkonstruktivistische Konzepte, wie am Beispiel Werlen diskutiert, einfach übernommen werden. Deutlich wird vielmehr die spannungsreiche theoretische Gemengelage in der aktuellen geographischen Forschung - und an eben diese muss, so wollen wir im Weiteren demonstrieren, eine geomedial gestützte Unterrichtspraxis anknüpfen.

\subsection{Möglichkeiten und Begrenztheiten von fach- lichen Raumkonzepten als Unterrichtsthema}

Der Einsatz von Geomedien eignet sich als methodischer Zugang zu Themen, die zu verschiedenen fachlichen Basiskonzepten vermitteln. Der Einbezug digitaler Geomedien in Unterrichtskontexte impliziert sowohl eine Auseinandersetzung mit dem absoluten, klassischen Raumkonzept als auch mit den multiscalaren Praxen: letztere sind technologische Grundbedingung dieser Medien, diese Medien reproduzieren die multiscalaren Praxen, sie sind aber auch unmittelbar anschlussfähig an distanzbasierte Präkonzepte, die wiederrum nicht mit sozial-konstruktivistischen Raumkonzepten in Einklang gebracht werden können. Die unterrichtspraktische Thematisierung fach- 
licher Raumkonzepte birgt demnach Widersprüchlichkeiten in sich, die selbst zum Unterrichtsthema gemacht werden sollten. Die Hypothese ist, dass Schüler/innen durch ihre Alltagserfahrung mit digitalen Geomedien bestens mit den Lage- und Distanzbeziehungen im Raum vertraut sind, digitale Geomedien zur Wiederbetonung des absoluten Raumkonzeptes beitragen, dass aber eine These "the meaning of space is produced via its communication" zu kurz greift (Thielmann et al. 2012, 21). Weiterhin nehmen wir an, dass Lage- und Distanzbeziehungen genauso Teil der Lebensrealität von Schülerinnen und Schülern wie das „Verschwinden der Ferne“ (Werlen 2015) durch die digitale Revolution sind, die konkrete Ausgestaltung dieser mehrdimensionalen Bezüge jedoch mit unterschiedlichen sozialen und kulturellen Ressourcen einhergeht. Die Gleichzeitigkeit dieser Erfahrung, also der multiscalare Raumbezug, sollte sich in Unterrichtsentwürfen zur Vermittlung von Raumkonzepten widerspiegeln, ohne dass von vornherein eine gleichartige Ressourcenausstattung angenommen wird.

Das sozial-ökologische Interaktionsmodell zeigt auf, dass einzelne Basiskonzepte wie „Raumkonstruktion und Raumkonzepte” meist in unmittelbarer Verbindung zu anderen Basiskonzepten, wie beispielsweise "Geoökosysteme“ oder „Mensch-Umwelt-Beziehungen" (Hinsch et al. 2014) stehen. Eine Auseinandersetzung mit einem einzelnen Basiskonzept im Unterricht kann unserer Meinung nicht zielführend sein. Explizit lässt sich hier außerdem zeigen, dass Basiskonzepte die Möglichkeit eröffnen könnten, eine fächerübergreifende Verbindung herzustellen. Genauso gut könnten die Basiskonzepte "Geoökosysteme“ oder „Mensch-Umwelt-Beziehungen“ im Lehrplan des Unterrichtsfaches Biologie und Umweltkunde zu finden sein. Dies könnte eine der zukünftigen Stärken von Basiskonzepten sein, zumal der fächerübergreifende Charakter durch eine gleiche Benennung für die Schüler/innen und Lehrer/innen greifbarer und konkreter gemacht werden könnte. Zugleich würde dies eine stärkere Kooperation und Koordination zwischen den Unterrichtsfächern bedingen. Auch Sander führt an, dass „[...] sich tragfähige Modelle für Basiskonzepte [...] nicht monodisziplinär entwickeln lassen" (Sander 2010, 59). Folgt man dieser Logik, kann nicht von den Basiskonzepten für das Unterrichtsfach Geographie und Wirtschaftskunde ausgegangen werden.

\section{Unterrichtsentwurf}

Unter Einbezug der bisherigen Überlegungen soll nun eine Unterrichtsskizze (siehe Anhang M1) Anregungen schaffen und aufzeigen, dass es möglich ist, im
Unterricht aktuelle Diskussionen innerhalb der Fachwissenschaften zum Thema zu machen, indem implizit auf die hier diskutierten Raumkonzepte Bezug genommen wird.

Ziel ist es nicht, dass Schüler/innen unterschiedliche Raumkonzepte namentlich kennen und erklären können, sondern dass sie durch praktische Arbeit mit GIS intuitiv die Begrenztheiten verschiedener Raumtheorien erfahren. Hier geht es auch darum, bei den Schülerinnen und Schülern ein Verständnis zu entwickeln, dass die Erklärungsansätze der Fachwissenschaften Lücken oder Unvereinbarkeiten mit von ihnen erlebten Realitäten aufweisen und sie ermuntert werden, diesen kritisch zu begegnen. Denn einer der größten Mängel im Bildungsbereich besteht unseres Erachtens darin, dass die im Unterricht vermittelten Theorien der Fachdisziplinen oftmals einen absoluten Wahrheitsanspruch vorgeben. Nicht zuletzt GIS selbst sind dafür teilweise ein Beispiel (Dodge et al. 2009; Schuurman 2004). Ohne Anspruch auf Vollständigkeit sei hier insbesondere verwiesen auf die Möglichkeiten und Gefahren, mit GIS unter Nichtbeachtung kartographischer Grundregeln Karten zu erstellen, dabei die Geo- und Attributdaten unkritisch in Bezug auf Datengüte, - aktualität, -maßstäblichkeit und ihr Aggregationsniveau zu analysieren ohne den Distanzdeterminismus von GIS zu hinterfragen und von der vermeintlichen Genauigkeit der computergestützten Analyse auf die Genauigkeit der zugrundeliegenden Daten zu schließen.

Um die Unterrichtseinheit ${ }^{6} \mathrm{zu}$ strukturieren, wird zu Beginn folgende Leitfrage gestellt: „Was kennzeichnet meinen Freizeitraum?" Ein weiteres Ziel derartiger Fokusfragen kann auch sein, die Präkonzepte der Schüler/innen zu eruieren, indem die Schüler/ innen zu Beginn einer Unterrichtseinheit versuchen, auf Grundlage ihrer Erfahrungen und ihres Vorwissens, die oben angeführte Frage schriftlich zu beantworten. Eine mündliche Beantwortung eignet sich weniger gut, da die Gefahr der Beeinflussung durch die Meinung der Mitschüler/innen besteht. Die Formulierung „mein“ wurde absichtlich gewählt, um den Bezug auf die Schüler/innen hervorzuheben und sie direkt anzusprechen. Interessant kann es auch sein, am Ende der Unterrichtseinheit die Fokusfrage erneut seitens der Schüler/innen beantworten zu lassen, um Konzept-Wechsel-Beziehungen sichtbar zu machen.

In einer ersten Arbeitsphase werden die Schüler/innen dazu angehalten, mit Hilfe digitaler Geomedien

\footnotetext{
Der ausgewählte Lehrplanbezug aus dem Lehrplanentwurf für die geplante Unterrichtseinheit wäre „die Konstruktionen von Räumen und raumbezogenen Identitäten zu untersuchen " (Hinsch et al. 2014, 61). Auch im aktuell gültigen Lehrplan für die Sek. II AHS der achten Klasse gibt es eine sehr ähnliche Formulierung, die diesem Lernziel entspricht (BMBF 2004).
} 
(beispielsweise OpenStreetMap oder Scribble Maps) und eines Arbeitsblattes, ihr Freizeitverhalten in einen von ihnen gewählten digitalen Kartenausschnitt einzutragen. Konkret wird hier auf den räumlichen Nahraum der Schüler/innen Bezug genommen. Einige Aktivitäten, wie Onlinespiele spielen, können nicht in die Karte eingetragen werden. Das dadurch entstehende Spannungsverhältnis lokalisierbarer und nicht lokalisierbarer Freizeitaktivitäten zeigt den Schüler/ innen auf, dass

- digitale Karten den räumlichen Alltag nur unzureichend abbilden können, da absolute Raumkonzepte nicht ausreichen, um die Lebenswirklichkeit wiederzugeben,

- aber gewisse Freizeitaktivitäten räumlich gebunden (z. B. Alpines Klettern) und durch Geofaktoren determiniert sind.

In einem weiteren Schritt werden die Karten anonymisiert und die Schüler/innen dazu angehalten, eine von ihren Mitschülerinnen und Mitschülern gestaltete Karte auszuwerten und zu kommentieren, also das Freizeitverhalten der Mitschüler/innen mithilfe der Karte wiederzugeben. Aufgrund des Unvermögens gewisse Aktivitäten räumlich zu fixieren, liefert die Karte ein verzerrtes Abbild des jeweiligen Freizeitverhaltens. Mit derartigen Unterrichtseinheiten können die Schüler/innen ein Bewusstsein für die Begrenztheit räumlicher Darstellungen entwickeln.

Eine weiterführende Diskussion im Plenum kann noch expliziter auf die unterschiedlichen Raumkonzepte Bezug nehmen. Mögliche Diskussionsinhalte könnten sein

- physisch konkrete Raumaneignung vs. virtuelle Raumaneignung: beispielsweise tatsächliches Skifahren vs. simuliertes Skifahrerlebnis mittels eines Computerspiels oder Surfen auf Hawaii vs. eine Sportübertragung der Surf-Weltmeisterschaft auf Hawaii. Laut Werlen (2015) sind wir durch das „Verschwinden der Ferne“ geprägt, d.h. die Relation von Nähe/Vertrautheit und Ferne/Unvertrautheit hebt sich auf (Werlen 2015, 90). In Frage gestellt werden sollte, ob eine konkrete leibliche Raumerfahrung einer in Form von digitalen Medien vermittelten Raumerfahrung gleichgesetzt werden kann.

- Verfügbarkeit und (regelmäßige) Zugänglichkeit zu Freizeiteinrichtungen im Nah- und Fernraum: zur Thematisierung der ungleichen Ressourcenverteilung zur Gestaltung von Räumlichkeiten und auch zur Überwindung absoluter räumlicher Distanzen; nicht zuletzt spiegelt sich die individuelle Ressourcenausstattung der Schüler/innen in ihrem Freizeitverhalten wider.
- Veränderung/Adaptierung des Naturraums durch/für Freizeitaktivitäten: zur Förderung des Verständnisses, dass materielle Realitäten raumzeitlich durch den Menschen verändert werden (Fischer-Kowalski \& Erb 2006, 37 ff.). Hier können durch Beispiele wie Wanderwege, Kletterrouten etc. Bezüge zum metrischen Raumkonzept hergestellt werden, insofern als auch "absolute“ Distanzen/Höhen überwunden werden müssen.

- Einfluss des Schulstandortes auf die Freizeit: beispielsweise durch lange Anfahrtszeiten

- $\quad \ldots$

Diese Verbindung des metrischen, absoluten und klassischen Raumkonzepts (den GIS inhärent) mit multiscalaren Perspektiven ermöglicht Schülerinnen und Schülern, ihre individuellen Raumvorstellungen zu erweitern und kritisch zu betrachten. In all diesen beispielhaften Anregungen zur Diskussion werden beide Raumkonzepte zur Geltung gebracht, aber auch deren jeweiligen Begrenztheiten aufgezeigt.

\section{Conclusio}

Anhand der Konzipierung des Basiskonzeptes „Raumkonstruktion und Raumkonzepte " haben wir grundlegende raumtheoretische Überlegungen angestellt und dabei prinzipielle Grenzen von GIS in Verbindung mit sozialkonstruktivistischen Theorieangeboten aufgezeigt. Damit dieses Basiskonzept unter Verwendung von GIS anschlussfähig gemacht werden kann, bedarf es aus unserer Perspektive auf der einen Seite einer Auseinandersetzung mit dem metrischen Raumkonzept, das auf das klassische absolute Raumkonzept zurückzuführen ist, und damit an ein System von Lage- und Distanzbeziehungen gebunden ist. Auf der anderen Seite müssen multiscalare Betrachtungsweisen einfließen, welche nicht unbedingt über die Anwendung von digitalen Medien oder Geomedien explizit gemacht werden können. Es gilt, auf die ungleiche Ressourcenausstattung zur Gestaltung von Räumen, aber auch auf die Fähigkeit der Distanzüberwindung zu verweisen. Eine virtuelle Distanzüberwindung oder Raumgestaltung kann nicht mit der physischen gleichgesetzt werden. Gelingt es im Rahmen des GWK-Unterrichts die vorgebrachten Kritikpunkte bei der Anwendung von GIS und digitalen Geomedien zu berücksichtigen, dann kann die geplante Implementierung des Basiskonzepts „Raumkonstruktion und Raumkonzepte" einen Mehrwehrt leisten. Prinzipiell ist das Basiskonzept dafür anschlussfähig, da es einen multiperspektivischen Zugang erlaubt, so dass insbesondere an vermutete Präkonzepte der Schüler/ innen angeschlossen und die Entwicklung eines kri- 
tischen Denkens vorangebracht werden kann. Auf diese Weise können, wie am Unterrichtsentwurf verdeutlicht, Lehr- und Lerninhalte über die Ebene der Reproduktion vorgegebenen Wissens hinausgehen und zur selbständigen Reflexion und Problemlösung anregen. Eine Konsequenz ist hierbei allerdings, wissenschaftliche Theorien als fundierte und begründete, aber trotzdem nicht absolute Sichtweisen darzustellen, deren absoluten Wahrheitsanspruch also zu bestreiten. Zwar ist eine solche Vorstellung von Wissenschaft vermutlich bei den Schülerinnen und Schülern verankert, allerdings verweist unsere inhaltliche und methodische Herangehensweise auf das Spannungsverhältnis von Realität und Theorie und sie zeigt, dass keine einzelne wissenschaftliche Sichtweise allein ausreicht, um die Komplexität der Welt erfassen zu können.

\section{Literatur}

Amelina, A., T. Faist, N. Glick Schiller, \& D.D. Nergiz (2012): Methodological Predicaments of Cross Border Studies. In: Amelina, A., D.D. Nergiz, T. Faist, \& N. Glick Schiller (Hrsg.): Beyond Methodological Nationalism. Research Methodologies for Cross-Border Studies. New York/Abingdon: Routledge, 1-19.

Beck, U. \& E. Grande (2004): Das kosmopolitische Europa. Frankfurt a. M.: Suhrkamp.

Belina, B. \& B. Michel (Hrsg.) (2007): Raumproduktion. Beiträge der „radical geography“ - eine Zwischenbilanz. Münster: Westfälisches Dampfboot.

$\mathrm{BMBF}$ - Bundesministerium für Bildung und Frauen (2004): Geographie und Wirtschaftskunde. https://www.bmbf.gv.at/schulen/unterricht/lp/lp_neu_ ahs_06_11858.pdf?4dzgm2 (24.01.16).

Brenner, N. (2004): New State Spaces. Urban Governance and the Rescaling of Statehood. Oxford: Oxford University Press.

Dodge, M., R. Kitchin \& M. Zook, (2009): How Does Software Make Space? Exploring Some Geographical Dimensions of Pervasive Computing and Software Studies. In: Environ Plan A 41 (6), 1283-1293.

Dürr, H. \& H. Zepp (2012): Geographie verstehen - Ein Lotsen- und Arbeitsbuch. Paderborn: UTB.

Egner, H. (2006) : Autopoiesis, Form und Beobachtung: moderne Systemtheorie und ihr möglicher Beitrag für eine Integration von Human- und Physiogeographie. In: Mitteilungen der Österreichischen Geographischen Gesellschaft 148, 92-108.

Fischer-Kowalski, M. \& K. Erb (2006) : Epistemologische und konzeptuelle Grundlagen der Sozialen Ökologie. In: Mitteilungen der Österreichischen Geographischen Gesellschaft 148, 33-56.

Goodchild, M.F. (2010): Twenty years of progress: GIScience in 2010. In: Journal of Spatial Information Science (1), 3-20.
Hellmuth, T. (2014): Historisch-politische Sinnbildung. Geschichte - Geschichtsdidaktik - politische Bildung. Schwalbach: Wochenschau.

Herod, A. (2010): Scale. London: Routledge.

Hinsch, S., H. Pichler, T. Jekel, L. Keller \& F. Baier (2014): Semestrierter Lehrplan AHS, Sekundarstufe II. Ergebnis der ministeriellen Arbeitsgruppe. In: GW-Unterricht 136, 51-61.

Hof, A. (2014): Schlüsselkompetenz raumbezogenes Denken. Mehrwert von Geoinformationstechnologien für die geographische Lehre. In: Haffer, S. \& C. Peter (Hrsg.): Herausforderungen in der Geographiedidaktik - Neue Medien, Kompetenzen, Leitbilder, Realbegegnungen. Aachen: Shaker (Gießener Geographische Manuskripte 8), 71-84.

Hürtgen, S. (2015): Transnationalisierung und Fragmentierung: Euro-Betriebsratshandeln als multiscalare Praxis. In: Pernicka, S. (Hrsg.): Horizontale Europäisierung im Feld der Arbeitsbeziehungen. Wiesbaden: VS-Verlag, 17-54.

Kern, H. (1994): Globalisierung und Regionalisierung bei industrieller Restrukturierung. In: Krumbein, W. (Hrsg.): Ökonomische und politische Netzwerke in der Region. Münster u. a.: Lit, 141-152.

Kisser, T. (2016): Mit Geocaching auf dem Weg zu einem verbesserten topologischen Raumverständnis. In: Kartographische Nachrichten 66 (1), 14-20.

Lange, N. (2013): Geoinformatik in Theorie und Praxis. 3. Aufl., Berlin [u.a.]: Springer.

Lippuner, R. (2010): Operative Geschlossenheit und strukturelle Kopplung. Zum Verhältnis von Gesellschaft und Umwelt aus systemtheoretischer Sicht. In: Geographische Zeitschrift 98 (4), 194-212.

Löw, M. (2001): Raumsoziologie. Frankfurt am Main: Suhrkamp Taschenbuch Wissenschaft.

Mehren M. \& R. Mehren (2015): Kompetenzorientiert Unterrichten aufgezeigt am Beispiel des Fachs Geographie. In: Bresges, A., B. Dilger, T. Hennemann, J. König, H. Lindner, A. Rohde \& D. Schmeinck (Hrsg.): Kompetenzen perspektivisch. Interdisziplinäre Impulse für die LehrerInnenbildung. Waxmann, Münster, 57-80.

Robertson, R. (1998): Glokalisierung: Homogenität und Heterogenität in Raum und Zeit. In: Beck, U. (Hrsg.): Perspektiven der Weltgesellschaft. Frankfurt a. M.: Suhrkamp, 192-220.

Sander, W. (2010): Wissen im kompetenzorientierten Unterricht - Konzepte, Basiskonzepte, Kontroversen in den gesellschaftswissenschaftlichen Fächern. In: zdg 1, 42-66.

Schulze, U. (2015): Digitale Geomedien und empirische Bildungsforschung: Ein systematischer Literaturreview zum Lernen mit Geographischen Informationssystemen. In: Zeitschrift für Didaktik der Gesellschaftswissenschaften (ZDG), Jahrgang 6 (2), 96-120.

Schuurman, N. (2004): Geographical information systems series. A short introduction. Malden: Blackwell Publishing (Short introductions to geography).

Siegmund, A. \& U. Michel (2013): Digitale Geomedien. In: Böhn, D. \& G. Obermaier (Hrsg.): Didaktische Impulse/Wörterbuch der Geographiedidaktik. Begriffe 
von A-Z. Braunschweig: Westermann Schulbuchverlag, $53-54$.

Sitte, C. (2004): Ein neuer LP GWK für die AHS-Oberstufe 2004. In: Wissenschaftliche Nachrichten Nr. 125, $45-50$.

Stieger, S. (2016): „Wer, wie, was ... wieso, weshalb, warum: arbeiten, produzieren und konsumieren wir?"“ Posterpräsentation gehalten auf der Tagung Zukunft Fachdidaktik GW 2016, am 21. April 2016 in Schlierbach, Österreich.

Swyngedouw, E. (2004): Globalisation or 'glocalisation'? Networks, territories and rescaling. In: Cambridge Review of International Affairs, 17 (1), 25-48.

Uhlenwinkel, A. (2013): Geographical Concepts als Strukturierungshilfe für den Geographieunterricht. Ein international erfolgreicher Weg zur Erlangung fachlicher Identität und gesellschaftlicher Relevanz. In: Geographie und ihre Didaktik (GuiD) 1, 18-43.

Wardenga, U. (2002): Alte und neue Raumkonzepte für den Geographieunterricht. In: geographie heute 200, $8-11$.

Wardenga, U. \& P. Weichhart (2006): Sozialökologische Interaktionsmodelle und Systemtheorien. Ansätze einer theoretischen Begründung integrativer Projekte in der Geographie? In: Mitteilungen der Österreichischen Geographischen Gesellschaft 148, 9-31.
Weichhart, P. (2011): Humanökologie. In: Gebhardt, H., R. Glaser, U. Radtke \& P. Reuber (Hrsg.): Geographie. Physische Geographie und Humangeographie. 2. Aufl., Heidelberg: Spektrum, 1088-1097.

Weidenhaus, G. (2015): Soziale Raumzeit. Frankfurt am Main: Suhrkamp Taschenbuch Wissenschaft.

Werlen, B. (2015): Praktiken der Welt-Bindung: gesellschaftliche Raumverhältnisse als trans-disziplinäres Forschungsfeld. In: Europa Regional 21.2013 (2015), 1-2, 83-92. http://nbn-resolving.de/urn:nbn:de:0168ssoar-430095 (15.04.2016).

Werlen, B. \& M. Weingarten (2013): Alltägliches Geographie-Machen, Regionalisierung und die Grenzen des Materialismus-Paradigmas. In: Brand, O., S. Dörhöfer \& P. Eser (Hrsg.): Die konflikthafte Konstitution der Region. Kultur, Politik, Ökonomie. Münster, 74-91.

Werlen, B. \& R. Lippuner (2011): Sozialgeographie. In: Gebhardt, H., R. Glaser, U. Radtke \& P. Reuber (Hrsg.): Geographie. Physische Geographie und Humangeographie. 2. Aufl., Heidelberg: Spektrum, 687-713.

Abbildung

Quelle der Abbildung: https://pixabay.com/de/skateboardpark-akrobatik-skateboard-1031218/ (03.06.2016). Gemeinfrei (Public Domain) entsprechend der Verzichtserklärung Creative Commons CC0. 


\section{M1: Arbeitsblatt zu eigenen Freizeitaktivitäten}

1. Verorte in der Karte jene Orte, an denen du deine Freizeitaktivitäten ausübst. Als Anhaltspunkte dienen dir folgende Vorschläge:

- diverse Sportarten

- sich mit Freund/innen treffen

- einkaufen

- Onlinespiele spielen

- ein Selfie verschicken

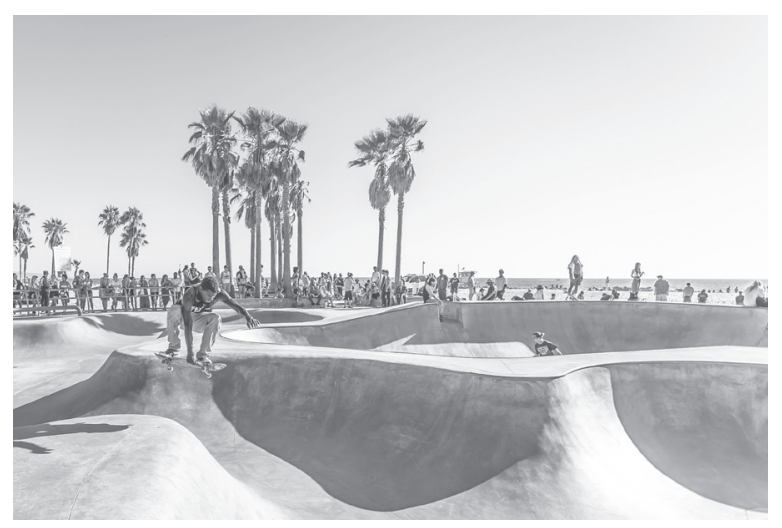

2. Wähle aus der Liste jene Aktivitäten aus, die deinen Präferenzen entsprechen und erweitere sie.

3. Nenne Freizeitaktivitäten, die du nicht in die Karte einzeichnen konntest und begründe diese Entscheidung.

4. Beschreibe die Lage der eingezeichneten Orte und versuche zu erklären, warum sie sich dort befinden. 